\title{
Tomographic color Schlieren refractive index mapping for computed axial lithography
}

\author{
Chi Chung Li \\ Department of Mechanical \\ Engineering, University of California, \\ Berkeley \\ ccli@berkeley.edu
}

\author{
Joseph Toombs \\ Department of Mechanical \\ Engineering, University of California, \\ Berkeley \\ jtoombs@berkeley.edu
}

\author{
Hayden Taylor \\ Department of Mechanical \\ Engineering, University of California, \\ Berkeley \\ hkt@berkeley.edu
}

\begin{abstract}
Computed axial lithography, when used in polymeric systems, directly solidifies freeform three-dimensional geometries inside liquid or gelled materials. Currently, this patterning system operates in open loop where projections are designed prior to the print so identification of errors and corrections can only be done after the printed object has been processed. This work introduces an in-situ 3D refractive index monitoring system to track localized material conversion by performing tomographic reconstruction from color Schlieren images. Our system successfully reconstructed evolving phase objects inside resins and the reconstruction quality was verified by comparison with isosurface laser scans. The technique provides support for physics-based real-time pattern modification to improve print fidelity and reduce manual iteration time when experimenting with new materials.
\end{abstract}

\section{CCS CONCEPTS}

- Hardware; • Emerging technologies; • Emerging optical and photonic technologies;

\section{KEYWORDS}

Computed axial lithography, color Schlieren, tomography, in-situ monitoring, phase contrast imaging

\section{ACM Reference Format:}

Chi Chung Li, Joseph Toombs, and Hayden Taylor. 2020. Tomographic color Schlieren refractive index mapping for computed axial lithography. In Symposium on Computational Fabrication (SCF '20), November 05, 06, 2020, Virtual Event, USA. ACM, New York, NY, USA, 7 pages. https://doi.org/10. $1145 / 3424630.3425421$

\section{INTRODUCTION}

Computed axial lithography (CAL) is a recently developed form of resin-based volumetric additive manufacturing (AM) [Kelly et al. 2019] inspired by the tomographic principles of computed axial tomography and intensity modulated radiation therapy [Schlegel et al. 2006]. In CAL, 2D images are projected through a rotating cylindrical volume of photosensitive material such that the cumulative

This work is licensed under a Creative Commons Attribution International 4.0 License.

SCF '20, November 05, 06, 2020, Virtual Event, USA

(C) 2020 Copyright held by the owner/author(s).

ACM ISBN 978-1-4503-8170-3/20/11.

https://doi.org/10.1145/3424630.3425421
$3 \mathrm{D}$ energy dose is sufficient to polymerize the design geometry. Because CAL is a layerless AM method, it is possible to print without supporting structures, to produce smooth surface finishes and to print in less than 10 minutes. In general, the photosensitive material has a high viscosity to limit sedimentation of the printed part during exposure and adequate transmission at the patterning wavelength. Currently, design of projections does not account for variability of photochemical responses of different resins. Interestingly, rotation during exposure also enables in situ tomographic 3D imaging of part formation which could provide material conversion feedback.

Sharpe et al. have developed a 3D imaging technique called optical projection tomography (OPT), used to probe 3D gene expression in biological samples, which bridges the gap between relatively lowresolution, large-scale methods like magnetic resonance imaging and micro-scale confocal fluorescence microscopy [Sharpe 2004]. However, the moderately high numerical aperture (NA) needed to resolve individual cells limits the depth of focus and samples must be in the range of 1-10 mm across. Improvements such as focal scanning via piezoelectric stages or electrically tunable lenses [Chen et al. 2014; Watson 2017] may be used to increase the imageable volume but may increase the projection acquisition time. Additionally, this method is only designed to detect the distribution of cells with fluorescent markers.

Tomographic imaging has previously been applied to volumetric AM [Loterie et al. 2020] to estimate local polymerization time and update dose prescription. Loterie et al. visualized photopolymerization with monochrome transmission imaging. Their system was assembled in a "focused" shadowgraphy [Settles 2001] configuration in which an expanded and collimated red laser was used as the light source. Shadowgraphy systems image the second spatial derivative of the refractive index (RI) or the gradient of the RI gradient. The local solidification time is determined by identifying the earliest time at which the corresponding volume element (voxel) appears to be darkened by more than an empirically-determined intensity threshold when observed from all angular projections. The local solidification time is then used to modulate the dose prescription to achieve simultaneous polymerization throughout the part. This technique only provides binary polymerization classification; thus information about the density and RI of the inhomogeneous region is inaccessible. By the design of this algorithm, solidification is not classified until a voxel appears to be darkened at all angles, leading to time inaccuracies and miscounting of voxels that are transparent at some views.

Schlieren imaging has long been an effective method for both qualitative assessment and quantitative analysis of fluid properties 
(i.e. pressure, density, and temperature fields) in a variety of scientific fields [Mercer 2003; Raffel 2015; Settles and Hargather 2017]. Several demonstrations have shown that Schlieren images processed with tomographic reconstruction techniques can produce a 3D map of RI field and subsequently pressure, density, etc [Agrawal et al. 1998; Décamp et al. 2008; Faris and Byer 1988; Greenberg et al. 1995; Srivastava 2013]. A continuous measurement of RI by tomographic Schlieren imaging would provide several benefits. It allows the progression of the polymerization process to be spatially and temporally tracked. Compared to binary threshold-crossing detection, continuous measurement directly quantifies the local error in degree of conversion, which is useful for real-time projection correction. Additionally, the time derivative of conversion can be estimated at any location. Finally, the high sensitivity of the Schlieren imaging also enables detection of undesirable background conversion of the resin precursor surrounding the target geometry.

In this paper, a color Schlieren imaging system is constructed to enable quantitative process feedback in CAL. First, an introduction to the mathematics of Schlieren imaging based on geometric optics is given and the theory of filtered backprojection for tomographic reconstruction from ray-deflection projections is presented. Then, the construction of an astigmatism-corrected Schlieren imaging system and color filter that encodes transverse RI gradient are detailed. Finally, the algorithm for computational reconstruction is described and accuracies of estimated print geometries are evaluated by dimensional comparison with printed components.

\section{THEORY}

\subsection{Schlieren Theory}

Conventional Schlieren imaging, as introduced by Töpler [Töpler 1867], is performed by introducing a knife-edge cutoff in the Fourier plane as a frequency cutoff in the imaging device (which is at the conjugate plane of the source). In the resultant $2 \mathrm{D}$ image, this filtering action introduces intensity variations which correspond to the RI inhomogeneities in the test area.

The following ray deflection analysis is derived from geometric ray optics with paraxial approximations and most relevant equations are reproduced from Settles [Settles 2001] and Agrawal et al. [Agrawal et al. 1998]. In general, when an undisturbed ray traveling along the $s$-axis (Figure 1A) encounters a region with RI variation in the $t$-direction it is deflected according to:

$$
\varepsilon=\frac{1}{n_{0}} \int \frac{\partial n(t, s, z)}{\partial t} \partial s
$$

where $\varepsilon$ is the angular deflection about the $z$-axis relative to the positive $s$-axis, $n_{0}$ is the RI of the surrounding, and $\frac{\partial n}{\partial t}$ is the gradient of the RI in the $t$-direction within the $t$-s plane. Assuming a small deflection, where $\varepsilon \approx \tan \varepsilon$, the spatial displacement in the Fourier plane along the $t$-axis can be expressed as

$$
d=f \varepsilon
$$

where $f$ is the focal length of the Schlieren lens in a dual-field-lens arrangement.

The intensity distribution in the Fourier plane replicates that at the light source plane because these are conjugate planes. For an ideal point light source, displacement towards or away from the knife edge in the Fourier plane results in either complete cutoff or passage of deflected rays. In a real system, however, a source with finite extent is partially blocked by an amount that is proportional to the source image displacement relative to the knife edge. For a rectangular slit source, the relative intensity variation due to this displacement can be given as

$$
\frac{\Delta I}{I} \sim \frac{f}{a} \varepsilon
$$

where $a$ is the width of the unblocked portion of the source rectangle. Thus, the sensitivity of the system for a given RI inhomogeneity is dependent on both the focal length of the second field lens and the degree of cutoff of the source image [Settles 2001]. In place of a knife-edge, a neutral-density graded filter may be used for increased measurement range i.e. the degree of deflection or magnitude of the gradient of the RI, at the cost of reduced sensitivity. Similarly, a graded color filter may be used, as will be described in Section 3.1.

\subsection{Tomographic Reconstruction}

Tomographic reconstruction is the inverse problem of estimating a function from a set of angularly separated projections each containing line integrals through the function domain. In this case, the function to be reconstructed is the RI field and the line integrals take the form of Equation 4. The ray deflection angles are the projected variables to be observed.

In order to reconstruct the $3 \mathrm{D}$ field, the problem is reduced to reconstruction of $2 \mathrm{D}$ slices that can be assembled to approximate the $3 \mathrm{D}$ field. In this analysis, normals of these slices are parallel to the rotation axis ( $z$ axis) of the print volume. For a projection angle $\theta$, the $x-y$ coordinate system (fixed relative to the vial) is transformed to the $t-s$ coordinate system (fixed relative to the optical system). For a given $z$-slice, at projection angle $\theta$, the transverse angular deflection (angle relative to the $s$-axis), $\varepsilon_{\theta}$, then becomes

$$
\varepsilon_{\theta}(t)=\int_{-\infty}^{\infty} \frac{\partial \hat{n}(t, s)}{\partial t} \partial s
$$

where $\hat{n}=\frac{n-n_{0}}{n_{0}}$ is the normalized RI change from the background RI, $n_{0}$ ( $n_{0}$ is the RI of completely uncured resin precursor material), and $\frac{\partial \hat{n}}{\partial t}$ is the gradient of the normalized RI in the transverse $t$-axis. Assuming negligible attenuation and a straight optical path, the order of differentiation and integration may be changed:

$$
\varepsilon_{\theta}(t)=\frac{\partial}{\partial t} \int_{-\infty}^{\infty} \hat{n}(t, s) \partial s .
$$

If the ordinary line integral projection is

$$
p_{\theta}(t)=\int_{-\infty}^{\infty} \hat{n}(t, s) \partial s
$$

then the Fourier transform of Equation 5 can be written as

$$
E_{\theta}\left(f_{t}\right)=i 2 \pi f_{t} P_{\theta}\left(f_{t}\right) .
$$

When absorption is negligible, the RI field for the $z$-slice can be reconstructed from the Fourier transform of a set of ordinary projections, $P_{\theta}\left(f_{t}\right)$, where $\theta \in[0, \pi]$ [Kak et al. 2002]:

$$
\hat{n}(x, y)=\int_{0}^{\pi}\left[\int_{-\infty}^{\infty} P_{\theta}\left(f_{t}\right) \exp \left(i 2 \pi f_{t} t\right)\left|f_{t}\right| d f_{t}\right] d \theta
$$

where $\left|f_{t}\right|$ represents the frequency response of the filter applied to the projections such that the sum of the backprojections gives an estimate of $\hat{n}(x, y)$ which approaches an exact reconstruction as 


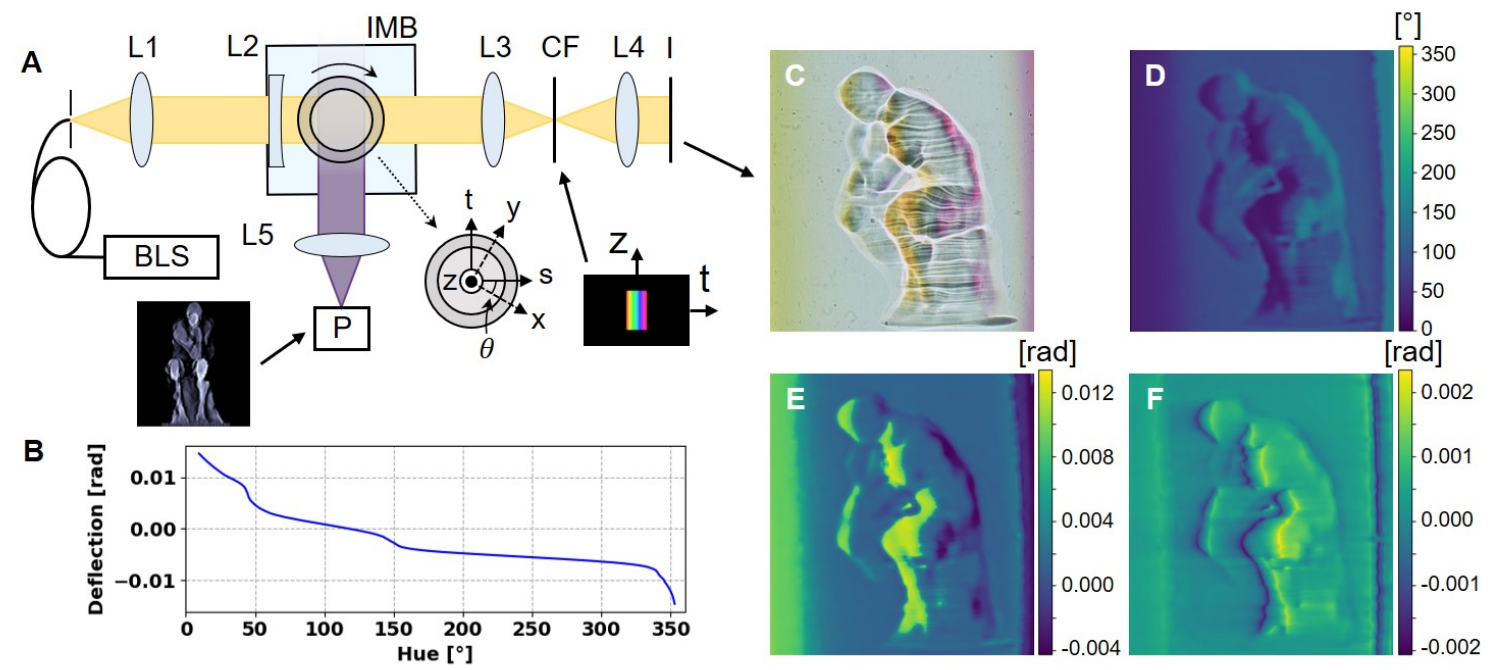

Figure 1: (A) Optical diagram of the CAL projection axis and orthogonal Schlieren imaging axis. L1 = collimating lens, L2 = weak concave cylindrical lens for astigmatism correction, $\mathrm{IMB}=$ index matching box, $\mathrm{L} 3=\mathrm{Schlieren}$ lens, $\mathrm{CF}=\mathrm{color}$ filter, L4 = focusing lens, I = image plane, $\mathrm{L} 5=$ collimating lens for projection, $\mathrm{BLS}=$ broadband white light source, $\mathrm{P}=$ projector (with an example of a projected image). (B) Deflection vs. hue curve generated from calibration with a planoconvex lens. (C) Color-filtered image captured during a CAL print. (D) Hue channel of (C). (E) Deflection calculated with calibration curve (B). (F) Final projection image after background deflection subtraction and convolution with filter.

sampling density is increased. Rearranging and substituting from Equation 7 and $t=-x \sin \theta+y \cos \theta$ gives

$$
\begin{aligned}
& \hat{n}(x, y)= \\
& -\frac{i}{2 \pi} \int_{0}^{\pi}\left\{\int_{-\infty}^{\infty} E_{\theta}\left(f_{t}\right) \exp \left[i 2 \pi f_{t}(-x \sin \theta+y \cos \theta)\right] \operatorname{sgn}\left(f_{t}\right) d f_{t}\right\} d \theta
\end{aligned}
$$

where $\operatorname{sgn}\left(f_{t}\right)=\left|f_{t}\right| / f_{t}$ is the sign function. The frequency response of the filter modified for reconstruction of ray deflection projections is $-i \operatorname{sgn}\left(f_{t}\right) / 2 \pi$ [Faris and Byer 1988].

\section{EXPERIMENTAL SETUP}

Figure $1 \mathrm{~A}$ shows a diagram of the optical setup. The Schlieren imaging system is positioned perpendicular to the CAL patterning light. A fiber-coupled broadband tungsten-halogen source (Thorlabs SLS201) is shaped to a slit source with metal blades at the output and used as the white light source. Identical $180 \mathrm{~mm}$ focal length (FL) achromatic doublet lenses (Thorlabs AC508-180-A-ML) are used as the Schlieren field lenses (L1 and L3). L3 is positioned one focal length away from the center of the vial. An acrylic box contains mineral oil $(n \approx 1.47)$ for approximate index matching the vial containing urethane dimethacrylate IPDI monomer (Esstech \# X-851-1066) ( $n=1.4938)$ with $0.25 \mathrm{M}$ concentration of Irgacure 907 photoinitiator. A cylindrical planoconcave lens (Thorlabs LK1900L1) is used to compensate further for astigmatism introduced by remaining index mismatch (L2). A $30 \mathrm{~mm}$ FL achromatic doublet lens is used as the focusing lens (L4) (Thorlabs AC254-030-A-ML). The camera used is a Panasonic GH4 body (without lens) set in $24 \mathrm{fps}$ and $3840 \times 2160$ pixel video capture mode.

\subsection{Color Filtering}

As an alternative to the knife edge of ordinary Schlieren imaging, a graded color filter can be used to encode the locations of rays in the Fourier plane into color instead of intensity. In this work, a 5 $\mathrm{mm} \times 20 \mathrm{~mm}$ linear-hue color filter is constructed by commercial photographic recording (Slides from Digital, Oakland, CA), where hue (of the hue-saturation-intensity (HSI) color space [Gonzalez et al. 2009]) varies from $0^{\circ}$ to $360^{\circ}$ linearly end-to-end, as shown in Figure $1 \mathrm{~A}$. With this filter, rays' $\varepsilon_{\theta}(t)$ deflections result in changes of hue observed in the Schlieren image. Together with a color image sensor, the shifts in color can be mapped back to ray deflections for tomographic reconstruction of the RI field.

Compared with traditional intensity-based ray deflection representations, adoption of an intensity-decoupled color representation could potentially make the index imaging system more robust against changes in scattering and achromatic absorbance [Greenberg et al. 1995]. Moreover, intensity information carried by light rays is not fully discarded in the color filtering process so the preserved achromatic absorbance information could be further extracted for other purposes (e.g. tomographic absorbance reconstruction). As a side benefit, the color-coding scheme also allows more sophisticated filters to be developed in the future to simultaneously encode vertical and horizontal ray deflections.

The half plane occupied by the knife edge in conventional Schlieren imaging represents the half plane of spatial frequencies and corresponding phase front directions removed from the Fourier plane. The gradient direction of the color filter resembles that of the knife edge counterpart, selecting the ray deflection direction to be observed. In Figure 1A, this normal direction is along $t$ and 
hence only ray deflections projected onto the $t$ direction are observed. Any ray deflections that are not directly aligned with $t$ will be observed with a lower sensitivity, following vector projection principles. The hue gradient on the filter determines the sensitivity of the deflection measurement. The lateral dimension of the color filter is chosen such that the maximum ray displacement caused by sample inhomogeneities is contained in the transmissive region.

Before the printing experiment is run, we first calibrate the deflection-hue transformation. It is typical in Schlieren systems to calibrate the intensity or hue transformation with a standardized target such as a weak lens of known focal length and index of refraction [Settles and Hargather 2017]. We follow this technique by placing a planoconvex cylindrical lens (Thorlabs LJ1874L1) near the center of the field-of-view and capturing an image of the color variation as a function transverse distance from the center of the lens. The deflection-hue calibration curve is generated by mapping the theoretical ray deflection calculated with Snell's law to the hue channel.

\section{COMPUTATIONAL RECONSTRUCTION}

The tomographic reconstruction procedure (Algorithm 1) begins after all video frames are captured. The expected resolution is 50 pixels/mm on the image plane. All video is captured at $24 \mathrm{fps}$. Depending on the rotation speed $\left(24^{\circ} \mathrm{S}\right.$ or $\left.12^{\circ} \mathrm{s}\right)$, there are 360 or 720 frames per rotation, and there are several rotations per fabricated component. A $15 \times 15$ pixel averaging filter was applied to raw captured frames to reduce their effect in the reconstructions. Each frame (projection) is converted to HSI with the OpenCV library, and the hue channel is extracted and transformed to ray deflection with the calibration curve obtained during setup. Then, if the frame is captured in the first rotation, it is saved and used later for background deflection subtraction. After this, the filter in Equation $9\left(-i \operatorname{sgn}\left(f_{t}\right) / 2 \pi\right)$ is applied in the frequency space. The results of these steps are shown for an example frame in Figure 1C-F. To obtain a series of time-sampled sinograms (projection set) for reconstructions, we evaluated three different time-segmentation approaches, which are described in Section 4.1 below. Finally, each set of projections in the $\theta$ range $[0,2 \pi)$ corresponding to a time point is backprojected in 3D with Astra Toolbox [Aarle et al. 2016; Palenstijn et al. 2011]. Projection data on $[0,2 \pi)$ is used instead of $[0, \pi)$ because of asymmetric hue changes induced by imperfect rotation axis concentricity of the vial fixturing. The result is saved as a $3 \mathrm{D}$ array in which each element contains the relative RI field change.

\subsection{Time-segmentation}

The projection images are taken at increasing time points and azimuthal angles, creating an imaging sequence that is helical in time-angle space, as shown in Figure 2A. For complete reconstruction, images have to be grouped into sets of $0-360^{\circ}$ projections. However, the polymerization state of the material evolves during any given revolution, so the method of grouping the projection data prior to reconstruction may affect temporal resolution and reconstruction quality. We therefore implemented and compared three possible time-segmentation methods with increasing timecorrection capability.

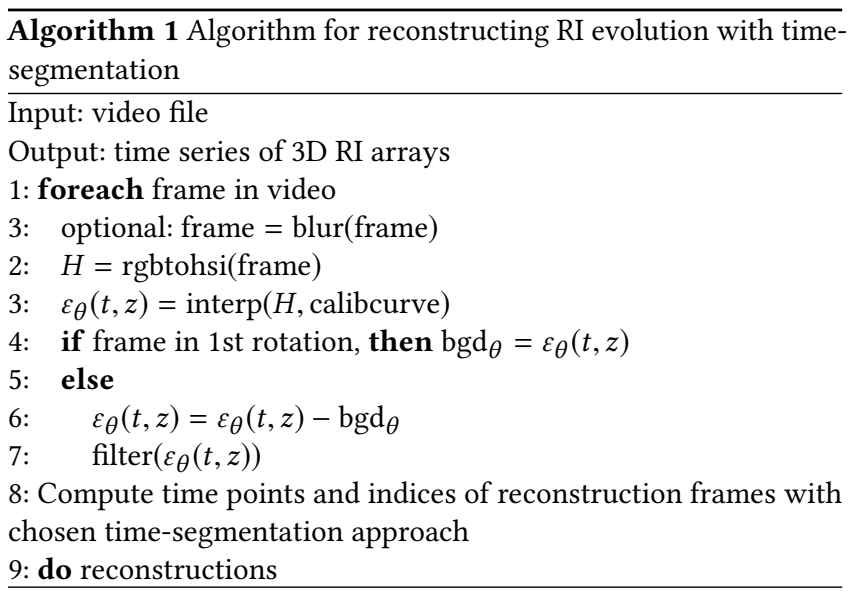

The first, naïve, approach to reconstruct the evolving RI field is to consider each $0-360^{\circ}$ set of projections as an independent window (IndW) of data. Because projections are not acquired simultaneously, each independent reconstruction actually represents the $\hat{n}$ field spanning the time of one rotation period centered in the middle of that period. As rotation rate and period are inversely proportional, the time resolution of the approach increases with increasing rotational velocity $\omega$. However, clearly this method cannot resolve events occurring on a time scale shorter than the window timespan and if higher temporal resolution is desired the frame rate of the imaging sensor must increase with $\omega$ in order to maintain high angular sampling rate $f_{\theta}$.

As an extension of the IndW approach, the moving window (MW) approach selects the subset of projection data for reconstruction at more finely resolved time. One reconstruction can be performed per frame instead of per rotation. Each forward time step is accomplished by adding the next acquired frame in the image sequence to the MW, removing the oldest frame in the MW $\left(f_{\theta}\right.$ remains constant), and reordering projections to maintain constant reconstruction orientation. The midpoint in time of the MW (Figure 2) is taken to be the timepoint of reconstruction. Consequently, there is a minimum delay of one half the window time span. With this technique, time resolution is similarly limited by the rotation period (window size) and the frame rate of the imaging sensor.

Kalender et al. introduced an approximate reconstruction technique for spiral projection acquisition geometry in which the human patient moves continuously through the continuously-rotating $\mathrm{x}$-ray scanner gantry. In this configuration, only one projection is acquired for any given slice orthogonal to the $z$-axis of the patient (which is also the axis of patient movement). Therefore, to estimate complete data for a virtual reconstruction $z$-slice, the missing projection data is estimated by a linear interpolation of adjacent scans acquired at the same angles weighted by the scans' distance from the virtual plane [Kalender et al. 1990; Schlegel et al. 2006; Shaw 2014]. In our final approach, we adopt a similar interpolation window (IntW) scheme but weighted instead by temporal distance because the imaging sequence is moving spirally through time instead of along the $z$-axis. 


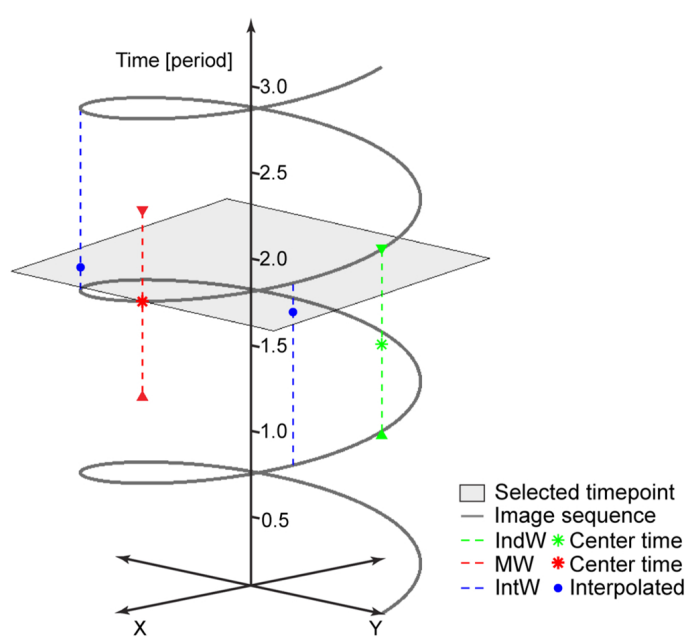

Figure 2: Visual representation of Schlieren-time segmentation geometry. Time is exchanged with the $z$-axis and a single projection frame corresponds to one point on the helix representing angular sampling as the resin vial rotates and time progresses. As projections are recorded with a single fixed camera only one angular projection can be acquired at a time. For IndW and MW, dashed lines represent the timespan of the window. For IntW, they connect the referenced projections.

First, we select a virtual time plane, the instantaneous time at which the RI field is to be reconstructed (Figure 3). Then, projection deflection data is interpolated onto this virtual time plane by referencing projections less than one period, $T$, before or after:

$$
\varepsilon_{\theta}(t, z)=(1-\sigma) \varepsilon_{\theta}(\tau, z)+\sigma \cdot \varepsilon_{\theta}(\tau+T, z)
$$

where $\varepsilon_{\theta}(t, z)$ is the estimated virtual projection data at time $t$ and angle $\theta, \tau$ is the latest time (before $t$ ) at which a projection is acquired for angle $\theta, \varepsilon_{\theta}(\tau, z)$ and $\varepsilon_{\theta}(\tau+T, z)$ are the previous and next projections (in time) acquired at the desired angle $\theta$. The weighting factor $\sigma$ is calculated as $\sigma=(t-\tau) / T$.

\section{PRINT AND RECONSTRUCTION RESULTS}

Three reconstructions (Figure $3 \mathrm{~A}-\mathrm{C}$ ) are created using an image sequence with 180 frames per vial rotation captured during a print of length 455 seconds and $12{ }^{\circ}$ s rotational velocity at a requested reconstruction time of 355 seconds. Figure $3 \mathrm{~A}$ is a reconstruction slice of the nearest rotation of the print (from 330-360 s) generated with the IndW approach. Figure $3 \mathrm{~B}$ is a slice generated with a MW with center frame at 355 seconds (from $340-370$ s). Figure $3 \mathrm{C}$ is a slice generated with an IntW centered at the same time (referencing frames from (325-385 s). The face and vertex structured stereolithography (.stl) file which is used as the starting point of the projection design algorithm is shown in Figure 3D and F, alongside laser scans (Romer RA-7525 SI) of the printed parts and isosurfaces that were extracted from the IntW-reconstructed $\hat{n}$ field near the end of the printing processes. Each isosurface is obtained with the marching cubes algorithm of the Python scikit-image image processing library [Lewiner et al. 2003; Walt et al. 2014.] with an isovalue of $\hat{n}=4 \times 10^{-4}$ (D) and $\hat{n}=2 \times 10^{-4}$ (F). The source of roughness on the scanned part surfaces could potentially be discretization errors from faceting point clouds, while the voxel-scale roughness on the isosurface results from the marching cube algorithm. The histograms in Figure $3 \mathrm{E}$ and $\mathrm{G}$ are created by measuring the orthogonal projection length of isosurface vertices to the nearest triangles of the scanned mesh using the cloud-to-mesh tool in CloudCompare [Girardeau-Montaut 2020]. With current hardware (Nvidia GTX 1050Ti and Ryzen $16003.4 \mathrm{GHz}$ ) images are processed at $28 \mathrm{fps}$ and reconstruction of a $600 \times 600 \times 560$ voxel volume requires $6 \mathrm{~s}$.

In Figure 4 the time progression of a print of length 650 seconds and $24^{\circ} \mathrm{S}$ rotational velocity is depicted in captured video frames and reconstructions using the IntW method. In frames at later times, strong horizontal striations are observed. These are attributed to the self-focusing of light that occurs due to non-simultaneous resin gelation. The averaging filter is applied to reduce their effect in the reconstructions.

\section{DISCUSSION}

In the comparison of various time-segmentation techniques, there are significant differences in reconstruction quality. Reconstructions generated by IndW showed lower maximum $\hat{n}$ and lower reconstruction-to-background contrast. This outcome is driven by the fact that this naïve approach provides only reconstructions centered at the middle of each rotation. Thus, in general, there is a time difference between the arbitrary requested time point and the center frame of the nearest reconstruction (see Figure 2 for an illustration of the discrepancy). In this example, the center frame of IndW reconstruction lags those from other two methods by $10 \mathrm{~s}$ (1/3 period). Because the RI gradient strengthens over time due to polymerization, the $\hat{n}$ magnitude is underestimated.

With the MW approach, the reconstruction can be adequately centered around the requested time (355 s) because the nearest frame, instead of the nearest rotation, is selected as the center. However, compared to reconstruction with the IntW method, the MW approach introduces an azimuthal bias to the reconstructed RI cross-sections, as seen from the slightly darker upper-left and brighter bottom-right regions in Figure 3B. This bias is a consequence of the fact that projections are acquired at increasing times (and angles), while the RI gradient is simultaneously increasing during the printing process.

Of the three time-segmentation methods evaluated, IntW is believed to create the most accurate and uniform results because it references two-periods-worth of frames for each reconstruction and corrects for the temporal refractive index changes that occur. With this method, the reconstruction can be centered at any moment in time instead of the nearest frame.

A comparison between the scanned mesh and the RI reconstruction reveals that the reconstruction error is the smallest at the helical surface $(\lesssim 0.3 \mathrm{~mm}$ in magnitude) and greatest at the helical edge $(<-$ $0.3 \mathrm{~mm}$ ), excluding localized noise at the top of model. The localized undershoot error on the edge might come from the 15-pixel-wide smoothing action applied. Optimization of the smoothing filter may 

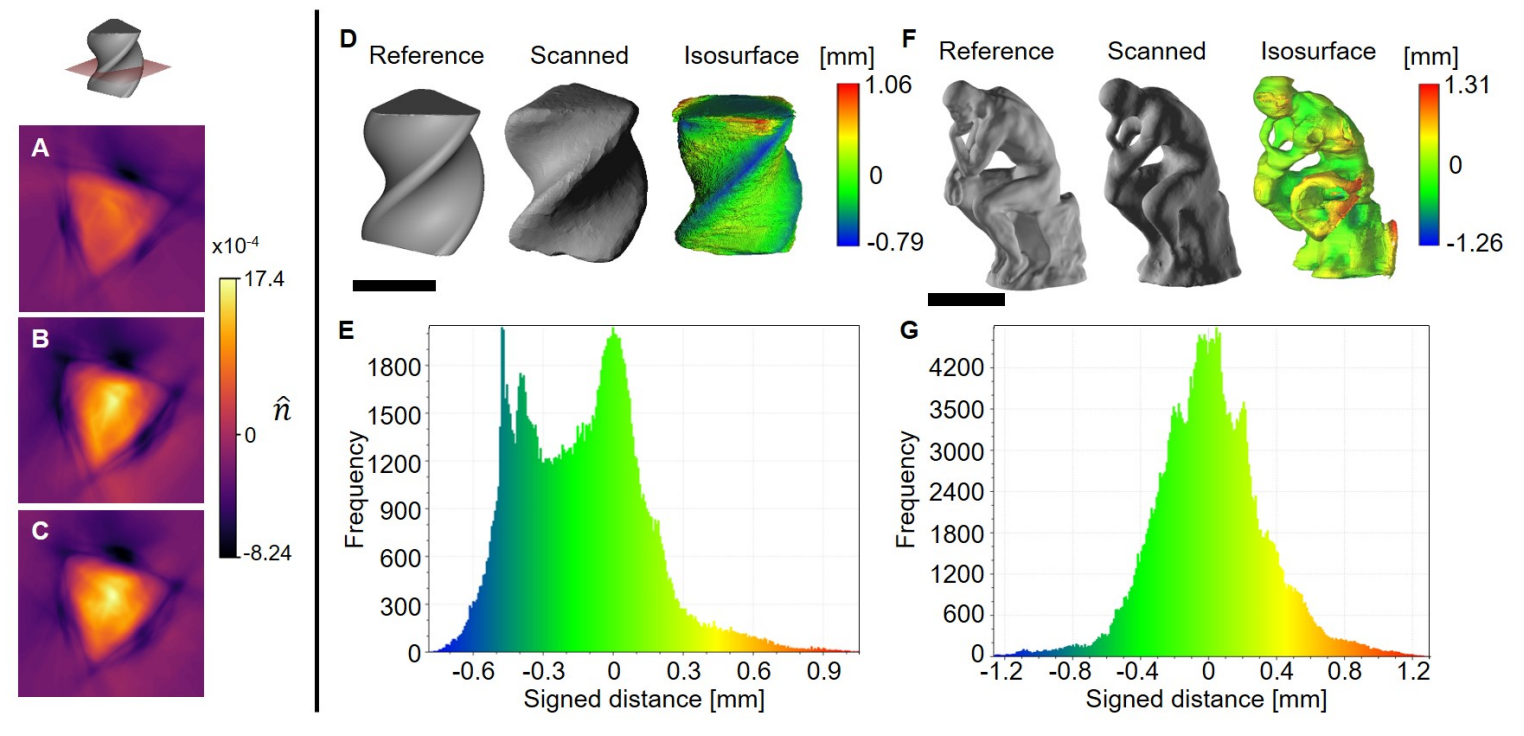

Figure 3: (A, B, C) Reconstructed $\hat{n}$ field slices using independent, moving, and interpolation window time-segmentation methods, respectively. (A) is reconstructed from $330-360 \mathrm{~s}$ (the $12^{\text {th }}$ rotation), (B) is MW centered at $355 \mathrm{~s}$ (from $\left.340-370 \mathrm{~s}\right)$ and (C) is IntW interpolated to $355 \mathrm{~s}$. (D) Triangle helix .stl file compared to laser-scanned printed part and the isosurface of isovalue $\hat{\boldsymbol{n}}=4 \times 10^{-4}$. (F) Thinker .stl file compared to laser-scanned printed part and the isosurface of isovalue $\hat{\boldsymbol{n}}=2 \times 10^{-4}$. (E, G) Histograms of signed distance of isosurface vertices in (D, F) respectively, from scanned meshes. (D)-(G) were obtained with the IntW method. Scale bars: $5 \mathrm{~mm}$.
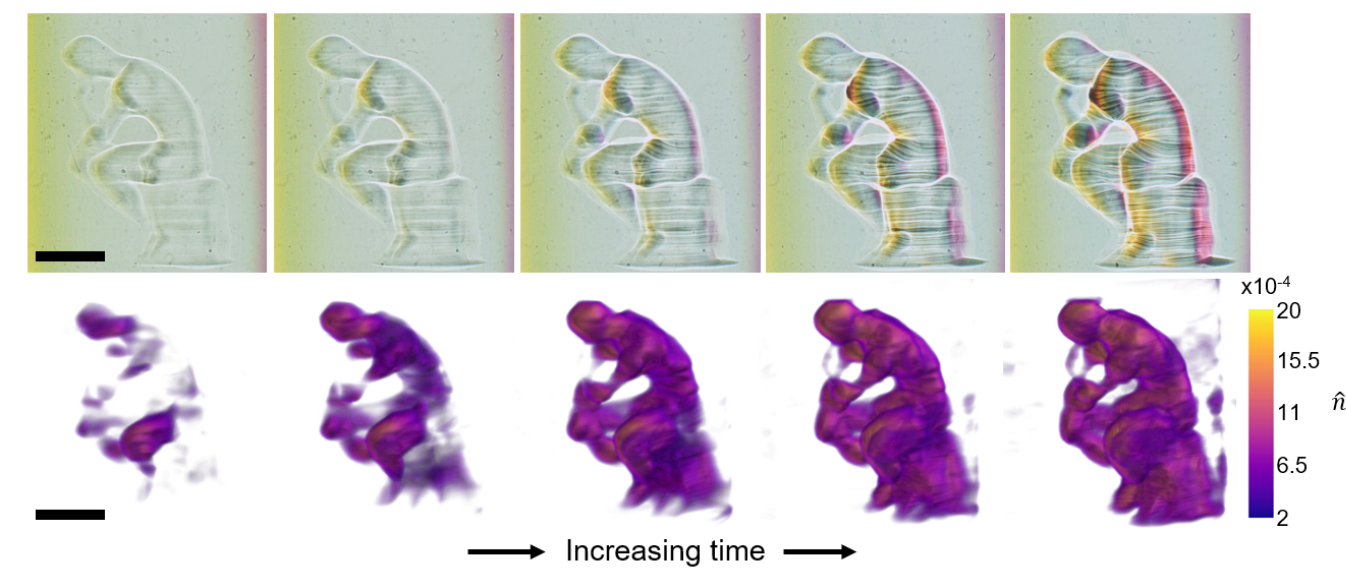

Figure 4: Top row: color Schlieren images captured during a CAL print of Rodin's 'Thinker'. Bottom row: corresponding reconstructed 3D $\hat{n}$ fields. Columns correspond to frames and reconstructions 510, 540, 570, 600, and 630 seconds, respectively. Scale bars: 5 mm.

improve the fidelity with which sharper edges can be reconstructed, while preserving the effectiveness of striation and noise removal.

In Figure 4, the fidelity of reconstruction can be qualitatively observed. At each time point, the topology of the $\hat{n}$ field closely resembles that suggested by the video frames. Figure $3 \mathrm{~A}-\mathrm{B}$ and Figure 4 indicate the absolute maximum normalized RI changes are on the order of $10^{-4}$. For instance, the maximum $\hat{n}$ predicted for the final reconstruction in Figure 4 is $20 \times 10^{-4}$, which corresponds to $n=1.4968(\Delta n=0.003)$. Although this RI change is seemingly rather weak, it is of the same order of magnitude as the values reported for UDMA-IPDI resin upon polymerization [Eom et al. 2011]. Quantitative verification of the result would require measurement of a standard target whose RI is both accurately known and close enough to that of the resin that all RI gradients remain within the measurement range when observed from any angle. For instance, the hue-calibration lens does not meet the latter criterion because 
at such RI, deflection is saturated when rays pass through a high transverse gradient along the planar side. This verification will also help to explain the negative index changes in Figure 3

The existence of non-uniform background hue (as shown in Figure 1C) is due to imperfect index matching and astigmatism correction. This background hue, although subtracted from projections, reduced the effective measurement range. Therefore, measurement saturation occurs earlier in regions where the background hue differs strongly from the center hue and prevented utilization of the full field of view.

The measured hue-deflection relationship is non-linear due to recording imperfections of the photographic filter and the unbalanced spectral distribution of the tungsten white light source. Although the deflection estimate remains accurate within the measurement range, the measurement sensitivity is shift-variant. We attempted to create filters that compensate for such non-linearity using the optimization method proposed by Greenberg et al. [Greenberg et al. 1995]. While linearity was improved over portions of the filter, we have not yet been able to achieve a linear deflection-hue relationship in the full $0-360^{\circ}$ hue range.

In these experiments, vial rotational eccentricity was reduced as much as possible with the current fixturing. However, it was not eliminated and could have reduced the sharpness of reconstructions. Without perfect index matching, eccentricity or 'wobble' results in a periodic lateral shift of the source image on the filter which leads to the mentioned undesirable background hue and sensitivity changes dependent on azimuth angle. With improved fixturing, these challenges could be reduced. Furthermore, time-segmentation windows could be shortened to half of their current respective lengths by employing the $180^{\circ}$ projection symmetry assumption $\left(p_{\theta}(t)=p_{\theta \pm \pi}(-t)\right)$, such that faster material transformation kinetics could be captured.

\section{CONCLUSION}

The results of this work can support the development of a realtime feedback CAL system where the projection images could be corrected during printing to: (1) reduce manual iterative experimentation for materials with unknown contrast and gelation threshold, and (2) induce a uniform degree of cure for simultaneous gelation throughout the printed object, which would have significant implications for print fidelity. Also, the printing process could be terminated automatically based on a specified or machine learned material-specific $\Delta n$, preventing overcuring and inaccurate part dimensions. Practically, real-time integration could be achieved with asynchronous image input/processing and reconstruction, all on faster computing hardware, within the time of a fraction of single rotation (currently $\sim 1 / 3 \mathrm{~T}$ for fastest $\omega$ ).

\section{ACKNOWLEDGMENTS}

This research is supported in part by the National Science Foundation under Cooperative Agreement No. EEC-1160494. We wish to acknowledge the staff of the UCB Mechanical Engineering Department Machine Shop, in particular Katherine Hom, for their help in laser scanning printed parts, and Prof. Vivek Subramanian's group in the UCB EECS department for providing the white light source.

\section{REFERENCES}

Wim van Aarle, Willem Jan Palenstijn, Jeroen Cant, Eline Janssens, Folkert Bleichrodt, Andrei Dabravolski, Jan De Beenhouwer, K Joost Batenburg, and Jan Sijbers. 2016. Fast and flexible X-ray tomography using the ASTRA toolbox. Optics Express 24, 22: 25129. https://doi.org/10.1364/OE.24.025129

Ajay K. Agrawal, Nelson K. Butuk, Subramanyam R. Gollahalli, and DeVon Griffin. 1998. Three-dimensional rainbow schlieren tomography of a temperature field in gas flows. Applied Optics 37, 3: 479. https://doi.org/10.1364/ao.37.000479

Lingling Chen, Sunil Kumar, Douglas Kelly, Natalie Andrews, Margaret J Dallman, Paul M. W. French, and James McGinty. 2014. Remote focal scanning optical projection tomography with an electrically tunable lens. Biomedical Optics Express 5, 10: 3367. https://doi.org/10.1364/BOE.5.003367

S. Décamp, C. Kozack, and B. R. Sutherland. 2008. Three-dimensional schlieren measurements using inverse tomography. Experiments in Fluids 44, 5: 747-758. https://doi.org/10.1007/s00348-007-0431-y

Ah-Hyang Eom, Duck-Su Kim, Soo-Hee Lee, Chang-Won Byun, Noh-Hoon Park, and Kyoung-Kyu Choi. 2011. Optical characteristics of resin composite before and after polymerization. Journal of Korean Academy of Conservative Dentistry 36, 3: 219. https://doi.org/10.5395/jkacd.2011.36.3.219

Gregory W Faris and Robert L Byer. 1988. Three-dimensional beam-deflection optical tomography of a supersonic jet. Applied Optics 27, 24: 5202. https://doi.org/10.1364/ AO.27.005202

Daniel Girardeau-Montaut. 2020. CloudCompare (version 2.6) [GPL software]. Retrieved from http://www.cloudcompare.org/

Rafael C. Gonzalez, Richard E. Woods, and Barry R. Masters. 2009. Digital Image Processing, Third Edition. https://doi.org/10.1117/1.3115362

Paul S. Greenberg, Robert B. Klimek, and Donald R. Buchele. 1995. Quantitative rainbow schlieren deflectometry. Applied Optics 34, 19: 3810. https://doi.org/10.1364/ao.34. 003810

Avinash C. Kak, Malcolm Slaney, and Ge Wang. 2002. Principles of Computerized Tomographic Imaging. Medical Physics 29, 1: 107-107. https://doi.org/10.1118/1. 1455742

W A Kalender, W Seissler, E Klotz, and P Vock. 1990. Spiral volumetric CT with singlebreath-hold technique, continuous transport, and continuous scanner rotation. Radiology 176, 1: 181-183. https://doi.org/10.1148/radiology.176.1.2353088

Brett E. Kelly, Indrasen Bhattacharya, Hossein Heidari, Maxim Shusteff, Christopher M. Spadaccini, and Hayden K. Taylor. 2019. Volumetric additive manufacturing via tomographic reconstruction. Science 363, 6431: 1075-1079. https://doi.org/10.1126/ science.aau7114

Thomas Lewiner, Hélio Lopes, Antônio Wilson Vieira, and Geovan Tavares. 2003. Efficient Implementation of Marching Cubes' Cases with Topological Guarantees. Journal of Graphics Tools 8, 2:1-15. https://doi.org/10.1080/10867651.2003.10487582

Damien Loterie, Paul Delrot, and Christophe Moser. 2020. High-resolution tomographic volumetric additive manufacturing. Nature Communications 11, 1: 852. https://doi. org/10.1038/s41467-020-14630-4

Carolyn R. Mercer (ed.). 2003. Optical Metrology for Fluids, Combustion and Solids. Springer US, Boston, MA. https://doi.org/10.1007/978-1-4757-3777-6

W.J. Palenstijn, K.J. Batenburg, and J. Sijbers. 2011. Performance improvements for iterative electron tomography reconstruction using graphics processing units (GPUs). Journal of Structural Biology 176, 2: 250-253. https://doi.org/10.1016/j.jsb.2011.07. 017

Markus Raffel. 2015. Background-oriented schlieren (BOS) techniques. Experiments in Fluids 56, 3: 60. https://doi.org/10.1007/s00348-015-1927-5

W. Schlegel, T. Bortfeld, and A.-L. Grosu. 2006. New Technologies in Radiation Oncology. Springer Berlin Heidelberg, Berlin, Heidelberg.

Gary S. Settles. 2001. Schlieren and Shadowgraph Techniques. Springer Berlin Heidelberg, Berlin, Heidelberg. https://doi.org/10.1007/978-3-642-56640-0

Gary S. Settles and Michael J. Hargather. 2017. A review of recent developments in schlieren and shadowgraph techniques. Measurement Science and Technology 28, 4. https://doi.org/10.1088/1361-6501/aa5748

James Sharpe. 2004. Optical Projection Tomography. Annual Review of Biomedical Engineering 6, 1: 209-228. https://doi.org/10.1146/annurev.bioeng.6.040803.140210

Chris C. Shaw. 2014. Cone Beam Computed Tomography. Taylor \& Francis, Boca Raton, Florida. https://doi.org/10.1016/B978-1-4377-0867-7.00070-3

Atul Srivastava. 2013. Development and application of color schlieren technique for investigation of three-dimensional concentration field. Journal of Crystal Growth 383: 131-139. https://doi.org/10.1016/j.jcrysgro.2013.09.001

A. Töpler. 1867. Optische Studien nach der Methode der Schlierenbeobachtung. Annalen der Physik und Chemie 207, 6: 180-215. https://doi.org/10.1002/andp. 18672070603

Stéfan Van Der Walt, Johannes L. Schönberger, Juan Nunez-Iglesias, François Boulogne, Joshua D. Warner, Neil Yager, Emmanuelle Gouillart, and Tony Yu. 2014. Scikitimage: Image processing in python. PeerJ 2014, 1: 1-18. https://doi.org/10.7717/ peerj.453

Thomas Watson. 2017. Advances In optical projection tomography. Imperial College London. Retrieved from All Papers/W/Watson - Advances In optical projection tomography.pdf 